\title{
MODELING OF MAGNETIC LABEL FIELD SENSING BY GMR STRUCTURE
}

\author{
Kirill Bukunov ${ }^{1,2}$, Georgy Babaizev ${ }^{1,2, *}$, and Nikolay Chechenin ${ }^{1,2}$ \\ ${ }^{1}$ Skobeltsyn Institute of Nuclear Physics, Lomonosov Moscow State University, Leninskie Gory, 1/2, 119991 Moscow, Russia \\ ${ }^{2}$ Faculty of Physics, Lomonosov Moscow State University, Leninskie Gory,1/2, 119991 Moscow, Russia
}

\begin{abstract}
An analytical model is developed to calculate 3D spatial distribution of vector magnetic field $\boldsymbol{H}(\boldsymbol{r})$ around a reference magnetic label of an arbitrary shape. A reliable method of $\boldsymbol{H}(\boldsymbol{r})$ modelling is important for location and precise positioning of a labelled object using GMR-based magnetic field sensors.
\end{abstract}

\section{Introduction}

Giant Magnetoresistance (GMR) sensors are widely used in countless applications, where sensors of linear or rotation motion are ones of them [1]. The main idea is a sensing of the magnetic field created by a reference magnetic label, which is normally a kind of permanent magnet placed on the moving part to be localized or positioned. The configuration of the field of the label is important to know. The magnitude and orientation of the magnetic field vector $\boldsymbol{H}(\boldsymbol{r})$ determines the sensing ability of GMR sensor placed at a remote point $\boldsymbol{r}$ with respect to the reference label position. For precise positioning and displacement sensing, a reliable method of modelling of magnetic field spatial configuration, produced by the reference labels, is highly important. Here we report on our method of calculation of the spatial configuration of magnetic field produced by a reference magnetic label.

\section{The model}

Considering the problem of magnetostatics to determine the spatial configuration of stationary magnetic field around the magnetic label at absence of electric currents, we can write Maxwell's equations together with additional equations closing the system in SI system as:

$$
\begin{gathered}
{[\boldsymbol{\nabla} \times \boldsymbol{H}]=0} \\
(\boldsymbol{\nabla} \cdot \boldsymbol{B})=0 \\
\boldsymbol{B}=\mu_{0}(\boldsymbol{H}+\boldsymbol{M})
\end{gathered}
$$

where $\boldsymbol{\nabla}$ - the Helmholtz operator, $\boldsymbol{B}=\boldsymbol{B}(\boldsymbol{r}), \boldsymbol{H}=\boldsymbol{H}(\boldsymbol{r})-$ vectors of magnetic induction and magnetic field, respectively, $\boldsymbol{M}=\boldsymbol{M}(\boldsymbol{r})$ - the magnetization vector, $\mu_{0}-$ magnetic constant.

Eq. (1) gives right to introduce a scalar magnetic potential $\phi=\phi(r)$ which satisfy the condition:

$$
\boldsymbol{H}=-\nabla \phi
$$

Substituting (3) into (2) and using (4), we obtain the Poisson equation for the scalar potential:

$$
\Delta \phi=(\nabla \cdot \mathbf{M}),
$$

where $\Delta$ is the Laplace operator. We denote the spatial region of localization of the magnetic substance (volume) as $\Omega$, and the surface bounding this volume as $\Sigma$. The problem arrives to find a sufficiently smooth function $\phi(\mathrm{r})$ in the outer region $r \in \Omega$, satisfying Eq. (5) with the given distribution $\boldsymbol{M}(\boldsymbol{r})$ and such that $\phi(\boldsymbol{r})$ $\underset{|r| \rightarrow \infty}{\longrightarrow} 0$. The solution of this problem exists, is unique and stable with respect to the input data. In general form the solution for the case of a uniform volume distribution of the magnetization is written via the Green's function of the Laplace operator:

$$
\phi(\boldsymbol{r})=-\frac{1}{4 \pi} \int \frac{\left(\boldsymbol{\nabla}^{\prime} * M(\boldsymbol{r})\right)}{\left|\boldsymbol{r}-\boldsymbol{r}^{\prime}\right|} d \boldsymbol{r}^{\prime}
$$

Applying the Ostrogradskii-Gauss theorem and taking into account the fact that the sources of the magnetic field are localized in the volume of the label and are absent at infinity, Eq. (6) transforms into:

$$
\phi(\boldsymbol{r})=\frac{1}{4 \pi} \iiint_{\Omega}\left(\boldsymbol{M}\left(\boldsymbol{r}^{\prime}\right) \cdot \boldsymbol{\nabla}^{\prime} \frac{1}{\left|\boldsymbol{r}-\boldsymbol{r}^{\prime}\right|}\right) d V^{\prime}
$$

To find the explicit form of the scalar potential $\phi(r)$ it is necessary to determine the choice of the coordinate system, and hence the choice of the shape of the magnetic label, since it will be associated with the symmetry of a certain coordinate system. Due to the fact that the mark should give sufficient amplitude for the magnetic field to detect the sensor, we choose a flat label shape, with a microsize in one spatial dimension and macro-dimensions for the other two. In order to simplify the model and not to take into account the evolution of the initial state of magnetization in time, we choose a sufficiently elongated shape of the label along one of the directions, thereby reducing the edge effects on the magnetization direction. We arrange the label, which is a rectangular parallelepiped with the sides $\Delta \mathrm{x}, \Delta \mathrm{y}, \Delta \mathrm{z}$ along the corresponding axes to the centre of the Cartesian coordinate system and with the ratio of the linear dimensions of the label $t: w: l=1: 10^{2}: 10^{3}$. We

* Corresponding author: kvyvg-george93@mail.ru 
consider the simplest case of the distribution $\boldsymbol{M}(\boldsymbol{r})$, which has an analytic solution - the case of a homogeneous magnetization over the entire volume of the label:

$$
\boldsymbol{M}=\left\{\begin{array}{lr}
M_{0} \boldsymbol{e}_{z}, & \boldsymbol{r} \in \Omega, \\
0, & \boldsymbol{r} \notin \Omega
\end{array}\right.
$$

Thus, we have

$$
\begin{array}{r}
\phi(\boldsymbol{r})=\frac{M_{0}}{4 \pi} \iiint_{\Omega}\left(\boldsymbol{e}_{\boldsymbol{z}} \cdot \boldsymbol{\nabla}^{\prime} \frac{1}{\left|\boldsymbol{r}-\boldsymbol{r}^{\prime}\right|}\right) d V^{\prime}= \\
\frac{M_{0}}{4 \pi} \iiint_{\Omega} \boldsymbol{d i v}\left(\frac{\boldsymbol{e}_{\boldsymbol{Z}}}{\left|\boldsymbol{r}-\boldsymbol{r}^{\prime}\right|}\right) d V^{\prime}=\frac{M_{0}}{4 \pi} \oiint_{\Sigma}\left(\frac{\boldsymbol{e}_{\boldsymbol{Z}} \boldsymbol{n}^{\prime}}{\left|\boldsymbol{r}-\boldsymbol{r}^{\prime}\right|}\right) d \sigma^{\prime}
\end{array}
$$

Here we have used the Ostrogradskii-Gauss theorem; $\boldsymbol{n}^{\prime}$ is the field of surface normals. The resulting expression must be integrated over the surface $\Sigma$ of a rectangular parallelepiped. Given that

$\left(\boldsymbol{e}_{\mathrm{z}} \boldsymbol{n}^{\prime}\right)=\left\{\begin{array}{cc}1, & \mathrm{z}=\frac{1}{2} \\ -1, & \mathrm{z}=-\frac{1}{2}\end{array} \quad\left\{\begin{array}{r}-\frac{\mathrm{t}}{2} \leq \mathrm{x} \leq \frac{\mathrm{t}}{2} \\ -\frac{\mathrm{w}}{2} \leq \mathrm{y} \leq \frac{\mathrm{w}}{2}\end{array}\right.\right.$

we obtain

$$
\begin{aligned}
& \phi(\boldsymbol{r})=\phi(x, y, z)= \\
& \frac{M_{0}}{4 \pi} \iint \frac{d x^{\prime} d y^{\prime}}{\sqrt{(x-x)^{2}+(y-y)^{2}+(z-z)^{2}}} \mid \begin{array}{c}
z^{\prime}=\frac{l}{2} \\
z^{\prime}=-\frac{l}{2}
\end{array}= \\
& \frac{M_{0}}{4 \pi} \int_{-\frac{t}{2}}^{\frac{t}{2}} d x^{\prime} \int_{-\frac{w}{2}}^{\frac{w}{2}} d y^{\prime}\left(\frac{1}{\sqrt{\left(x-x^{\prime}\right)^{2}+(y-y)^{2}+\left(z-\frac{l}{2}\right)^{2}}}-\right. \\
& \left.\quad \frac{1}{\sqrt{\left(x-x^{\prime}\right)^{2}+\left(y-y^{\prime}\right)^{2}+\left(z+\frac{l}{2}\right)^{2}}}\right)
\end{aligned}
$$

We introduce the function

$$
\begin{aligned}
& F(x, y, z) \stackrel{\operatorname{def}}{=} \iint \frac{d x d y}{\sqrt{x^{2}+y^{2}+z^{2}}}= \\
& -\operatorname{zarctg}\left(\frac{x y}{z \sqrt{x^{2}+y^{2}+z^{2}}}\right)+x \ln \left(y+\sqrt{x^{2}+y^{2}+z^{2}}\right)+ \\
& y \ln \left(x+\sqrt{x^{2}+y^{2}+z^{2}}\right)-x-y+z \operatorname{arctg}\left(\frac{x}{z}\right)+ \\
& \operatorname{zarctg}\left(\frac{y}{z}\right)+\text { const }
\end{aligned}
$$

It expresses the value of the indefinite integral (11) in terms of elementary functions. For this integral a substitution is performed in symmetric limits and the last five terms in (12) can be omitted, since they are odd functions for at least one argument and the constant term does not play a role. As a result, we obtain an explicit expression for the scalar potential of the magnetic field of the label, written in Cartesian coordinates through the function (12) with the omitted five last members:

$$
\begin{aligned}
& \phi(x, y, z)= \\
& \frac{M_{0}}{4 \pi}\left\{\begin{array}{l}
F\left(x-\frac{t}{2}, y-\frac{w}{2}, z\right)-F\left(x-\frac{t}{2}, y+\frac{w}{2}, z\right) \\
-F\left(x+\frac{t}{2}, y-\frac{w}{2}, z\right)+F\left(x+\frac{t}{2}, y+\frac{w}{2}, z\right)
\end{array}\right\} \mid \begin{array}{l}
z^{\prime}=\frac{l}{2} \\
z^{\prime}=-\frac{l}{2}
\end{array}
\end{aligned}
$$

To find the required magnetic field vector $\boldsymbol{H}=\left\{H_{x}, H_{y}\right.$, $\left.H_{z}\right\}$ we need to take the gradient from the scalar potential, as it is written in (1)

$$
\left\{\begin{array}{l}
H_{x}(x, y, z)=-\frac{\partial \phi(x, y, z)}{\partial x} \\
H_{y}(x, y, z)=-\frac{\partial \phi(x, y, z)}{\partial y} \\
H_{z}(x, y, z)=-\frac{\partial \phi(x, y, z)}{\partial z}
\end{array}\right.
$$

Expressions for each of the components have a complicated and cumbersome analytical form, so their graphic interpretation is presented below.

\section{Spatial configuration of magnetic field of particular reference label shapes}

Figure 1 shows a three-dimensional picture of the distribution of the magnetic field vector $\boldsymbol{H}$ in the form of discrete arrows regularly located in 3D space. The colour gradation reflects the modulus of the $\boldsymbol{H}$ vector at each particular point in space and the length of the arrows is

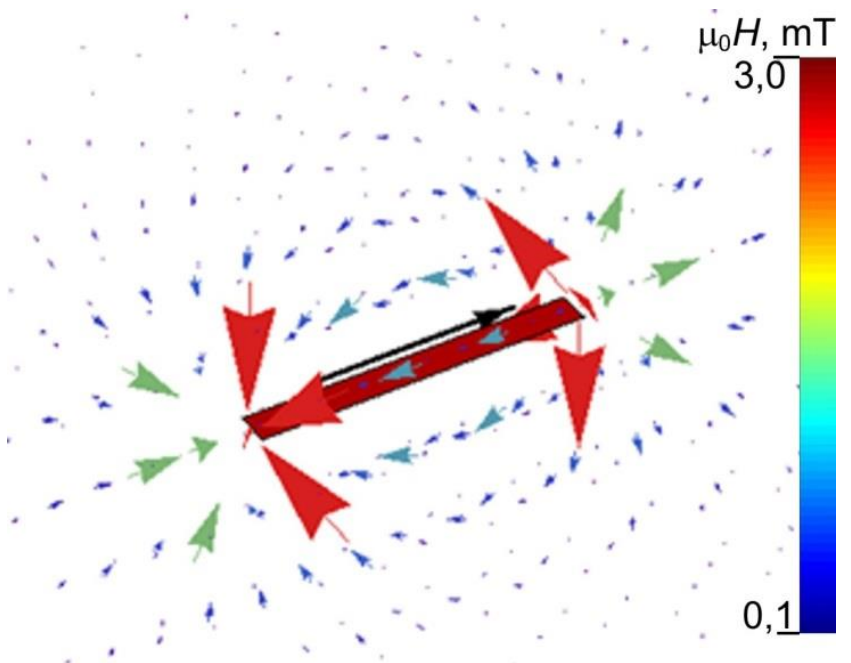

Fig. 1. 3D picture of $\boldsymbol{H}$ field configuration around the reference label.

proportional to the projection of $\boldsymbol{H}$ on the plane of drawing. Here and in the following figures the magnetic strip is shown in red. The black arrow above indicates the direction of magnetization (the positive direction of the $z$ axis). It is worth to note that the change in absolute label sizes does not affect the field configuration, but causes only a proportional change in the magnetic field strength.

In Figs. 2,3 distribution of components of magnetic field vector are over the $y$-z-plane with fixed $x$ coordinate $\left(x_{0}=1 \mathrm{~mm}\right)$. The third dimension of the plot is the projection of the magnetic field strength on a particular axis, $x$ (Fig. 2a) or $y$ (Fig. 3a). The spatial coordinates are normalized on the length of the label $(2$ $\mathrm{cm})$. 
The choice of the fixed coordinate $x$ perpendicular to the main surface of the label was made because the main relative label-sensor displacements are assumed in $y-z$ plane. It is obvious that the closer to zero the $x$ coordinate value, i.e. the closer the sensor set to the label, the stronger is the field.

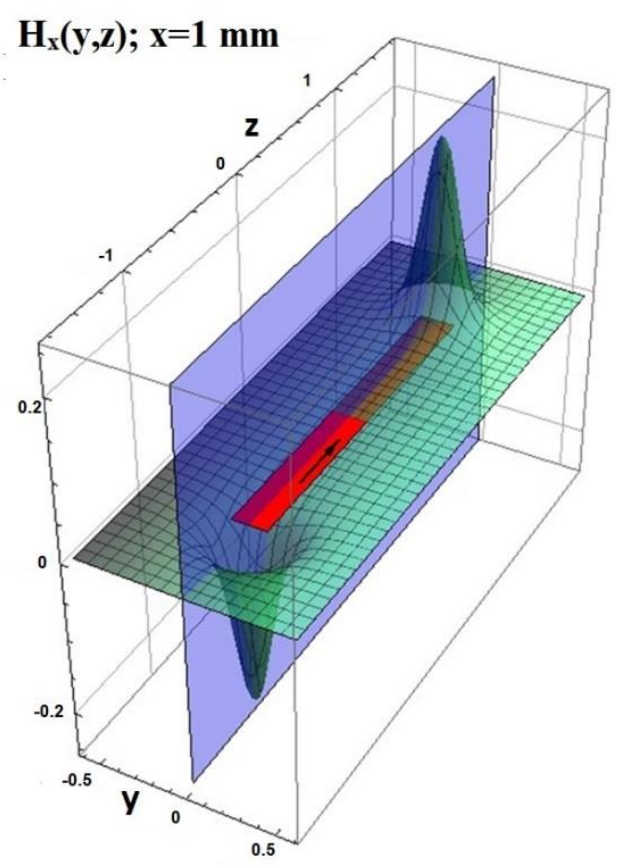

(a)

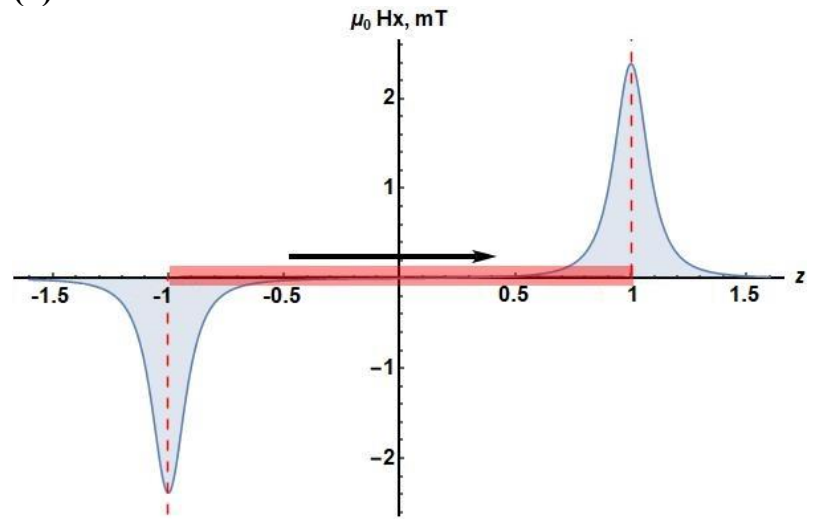

(b)

Fig.2. Distribution of $x$-component of the magnetic field $\mu_{0} \boldsymbol{H}_{\boldsymbol{x}}\left(x_{0}, y, z\right)$ at $x_{0}=1 \mathrm{~mm}$ (a) and cross-section of this distribution at $y=0(b)$.

The cross-sections of the field spatial configuration over the strongest field variations are shown in Figs. $2 b$ and $3 b, c$. The cross-section planes are highlighted in the same colour in Figs. 2a and $3 a$ as the plots in Figs. $2 b$ and $3 \mathrm{~b}, \mathrm{c}$. The numerical values of the components of vector $\boldsymbol{H}$ in the plots are given as multiplied by the magnetic constant and correspond to the components of magnetic induction in tesla, which coincide with magnetic field strength, Eq. (3), in the region outside the label where magnetisation is zero. The electromagnetic potential in Eq. (6 to 13) and, hence, the magnetic field strength depends on the uniform magnetization $M_{0}$ which was obtained as was obtained by:

$$
M_{0}=\alpha n \mu_{B},
$$

where $n$ is the concentration of ferromagnetic atoms in the volume, $\mu_{B}$ is the Bohr magneton, and $\alpha$ is a dimensionless empirical constant, indicating the number of Bohr magnetons per 1 atom of the magnetic substance. In our estimations we take $\alpha \cong 1$. In this case estimated field strength fits the experimental values for cobalt label. The magnetic field from a cobalt strip of 20 $\mu \mathrm{m} \times 2 \mathrm{~mm} \times 2 \mathrm{~cm}$ (thickness $\times$ width $\times$ length) at a
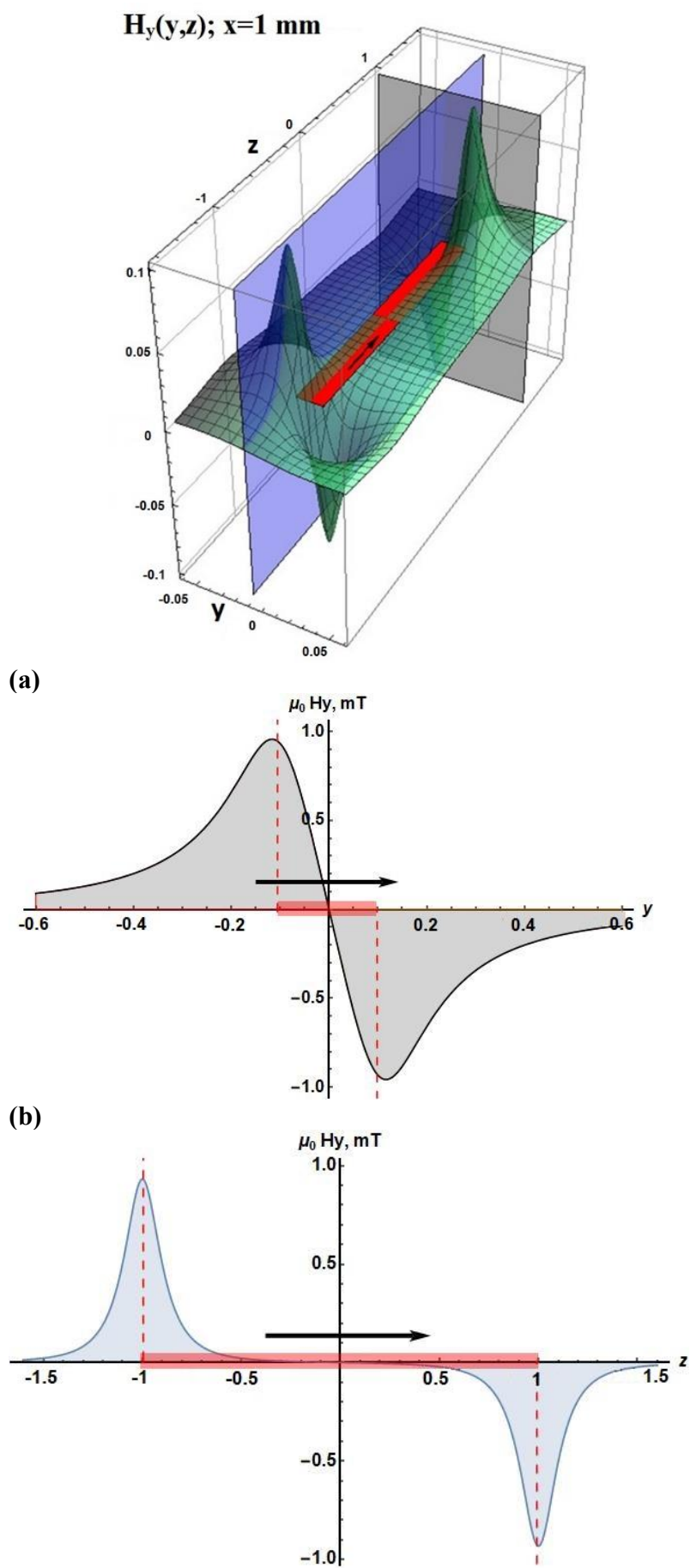

(c)

Fig.3. The distribution of the $y$-component of magnetic field $\boldsymbol{\mu}_{0} \boldsymbol{H}_{\boldsymbol{y}}\left(x_{0}, y, z\right)$ at $x_{0}=1 \mathrm{~mm}$ (a) and cross-section $\boldsymbol{\mu}_{0} \boldsymbol{H}_{\boldsymbol{y}}\left(x_{0}, y\right)$ of this distribution at $x_{0}=1 \mathrm{~mm}, z=l / 2(\mathrm{~b})$ and $\mu_{0} \boldsymbol{H}_{\boldsymbol{y}}\left(x_{0}, z\right)$ at $x_{0}=$ $1 \mathrm{~mm}, y=-w / 2$, (c).

distance of $1 \mathrm{~mm}$ in the region of the poles gives a field 
larger by order of magnitude than that of the Earth, which is about $50 \mu \mathrm{T}$.

One can note a common feature of the cross-sections in Figs. 2b, 3b,c: narrow peaks, in comparison with the size of the label, localized in the regions of the magnet poles. The collection of information about the magnetic field occurs only along the selected direction in space, therefore only the projections of the field strength for this selected direction are read. Of the possible combination of the field components $\left\{H_{x}(i), H_{y}(i)\right.$, $\left.H_{z}(i)\right\}$, where $i=x, y, z$, the choice was made in favor of $H_{z}(z)$, Fig. 4, since it was in this graph that several useful, in practical terms, properties are combined:

the presence of a "double drop" in the function graph in each constant-sign region of variation of its argument, this facilitates unambiguous determination of the position of the magnetic label relative to the sensor;

the field amplitude not diminishing to zero in the region between the poles: it also gives an advantage in the uniqueness of position determination;

a large gradient of the function in the direction of displacement (in areas close to the edges of the label): this improves the space resolution of the label location.

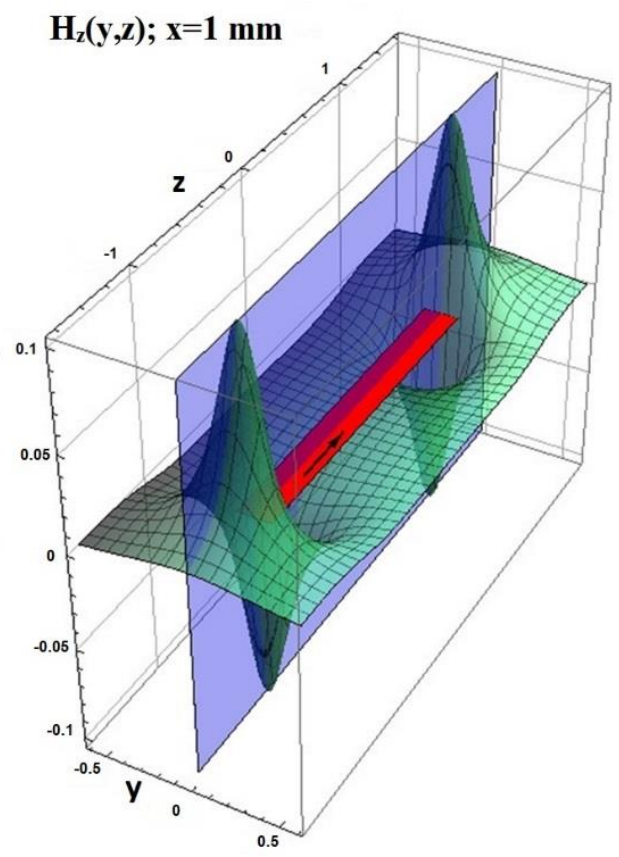

Fig. 4. The configuration of $z$-component of the field, $\mu_{0} H_{z}\left(x_{0}, y, z\right)$ at distant $x_{0}=1 \mathrm{~mm}$ from the magnetic label.

Based on the foregoing, it is possible to determine the relative arrangement of sensors in the sensor block, formulate the conditions for the minimum and maximum distances between the sensors, their number and give estimates of the positioning accuracy.

To follow the $H_{z}(z)$ component, as a possible solution, the sensor block can be a series of sensors in a row along the forward direction, which coincides with the magnetization direction in the label, that is, with the direction of its long side. The sensor unit should be positioned as close to the center of the label as possible to capture the maximum field amplitude. Obviously, one sensor in the sensor block will not be enough to uniquely identify the position in space over the field amplitude. The arrangement of the sensors in the row can be of regular or irregular spaced to optimize the accuracy of label displacement.

As an example, a detailed analysis which includes the mapping of the field derivative over the displacement direction, $\partial H_{z} / \partial z$, shows that for regular equidistant spacing along the label of $l=20 \mathrm{~mm}, 3$ sensors is enough to obtain the label positioning accuracy of $2 \mu \mathrm{m}$.

The magnetic field space distribution is used to estimate the effect of the field on the magnetoresistance of the spin-valve (SV) structure displaced by a certain distance $x_{0}$ from the magnetic stripe. The efficiency of the magnetoresistive sensing effect of the GMR SV structure strongly depends not only from the strength of magnetic field exerted, but also on magnetic properties ferromagnetic and antiferromagnetic layers, composing the SV. In a simple approximation, the component of the magnetic field from the reference magnetic label oriented at $90^{\circ}$ to the uniaxial anisotropy direction in the of the free layer in the SV structure rotates the magnetic moment by the angle [2]

$$
\varphi=\arccos \left(M_{\mathrm{F}} H_{\mathrm{F}} / 2 K_{u}\right),
$$

where $M_{\mathrm{F}}$ and $H_{\mathrm{F}}$ are magnetic moment and magnetic field, acting in the free layer of SV structure, and $K_{u}$ is the uniaxial anisotropy energy of the free ferromagnetic layer. The magnetoresistance of the $\mathrm{SV}$ in this case is determined from approximate relation

$$
R_{\mathrm{GMR}}=R_{\mathrm{GMR}}{ }^{0}(1-\cos (\varphi)) / 2
$$

Using the described technique we can estimate the signal response to variable field strength and, hence, sensitivity and space resolution of the device based on the array of GMR sensors to detect position and movement of the object.

\section{Conclusion}

An analytical approach is developed to calculate the $3 \mathrm{D}$ configuration of the magnetic field near the magnetic label. It allows modeling the response of the magnetic sensor or arrays of the sensors and optimization the sensor-to-sensor arrangement within an array of GMR sensors in order to increase the sensitivity and spatial resolution of the displacement transducer.

\section{References}

1. Reig C., Cardoso C., Mukhopadhyay S., Giant Magnetoresistance (GMR) Sensors. From Basis to State-of-the-Art Applications. (Springer-Verlag, Berlin Heideberg, 2013) 131p.

2. E. C. Stoner, E.P. Wohlfarth. Phil. Trans. Roy. Soc. (London) A240, 599 (1948) 